\title{
Temporomandibular joint reconstruction with vascularized free fibula flap following disarticulation resections: A protocol for a systematic review
}

\author{
Ahmed Abdelrehem
}

Funding: The author(s) received no specific funding for this work.

Potential competing interests: The author(s) declared that no potential competing interests exist.

\section{Abstract}

\begin{abstract}
Background: Mandible reconstruction using vascularized free fibula flaps (FFF) is now considered the state of the art, however, those including disarticulation resections and hence reconstruction of the temporomandibular joint (TMJ) are still challenging for most maxillofacial surgeons as the available literature reports are scarce. Most reports focused on the clinical outcomes, and few focused on the spatial deviations of the neocondyle. Several modifications and methods have been reported to support the fibula and improve the functional outcomes of the TMJ.

Objective: The aim of this systematic review is to investigate different functional and morphological outcomes following TMJ reconstruction with FFF after disarticulation resections and correlates with the different modification and factors.

Materials and Methods: An online search will be done to check all articles reporting TMJ reconstruction using FFF and advocating different outcomes. prospective clinical study was to introduce three modified techniques simultaneously with mandible reconstruction of the TMJ with vascularized free fibula flap (FFF) for dynamic mandible reconstruction and evaluate the different outcomes of such modifications in comparison to the conventional technique.
\end{abstract}

Temporomandibular joint reconstruction with vascularized free fibula flap following disarticulation resections: A systematic review

\section{Abstract}

Background: Mandible reconstruction using vascularized free fibula flaps (FFF) is now considered the state of the art, however, those including disarticulation resections and hence reconstruction of the temporomandibular joint (TMJ) are still challenging for most maxillofacial surgeons as the available literature reports are scarce. Most reports focused on the clinical outcomes, and few focused on the spatial deviations of the neocondyle. Several modifications and methods have been reported to support the fibula and improve the functional outcomes of the TMJ.

Objective: The aim of this systematic review is to investigate different functional and morphological 
outcomes following TMJ reconstruction with FFF after disarticulation resections and correlates with the different modification and factors.

Materials and Methods: An online search will be done to check all articles reporting TMJ reconstruction using FFF and advocating different outcomes. prospective clinical study was to introduce three modified techniques simultaneously with mandible reconstruction of the TMJ with vascularized free fibula flap (FFF) for dynamic mandible reconstruction and evaluate the different outcomes of such modifications in comparison to the conventional technique.

\section{Introduction}

Reconstruction of the temporomandibular joint (TMJ) for congenital or acquired deformities is a major challenge for maxillofacial surgeons. The absence of normal condylar three dimensional spatial positioning has the consequence of progressive and severe facial disharmonies. Successful reconstruction of the TMJ must reproduce the structures that are involved to restore their form and function and allow normal mandibular biomechanics (1-3)

Post oncologic resection defects of the mandible impair the form and function in addition to the esthetics. However, such defects have an impact on the temporomandibular joint and neocondyle, especially with disarticulation resections, including changes in the spatial positioning, biomechanics and joint morphology. This could be explained due to the loss of attachment of the masticatory muscles and ligaments which control the 3D positioning and biomechanics of the lower jaw and joint. In other words, the position of the neo-condyle and joint is mainly influenced by the extent of posterior mandible resection. Therefore, mandibular defects in the anterior region are often associated with less impact on the TMJ than those defects involving the ramus condyle complex. In case of disarticulation resection of the mandible, the transplanted neocondyle becomes passively positioned in the glenoid fossa lacking any form of support or anatomical anchorage due to loss of muscle and ligaments attachment without an anatomical anchor. As a result t the neocondyle is freely mobile and unsupported with resulting abnormal TMJ functions and deviations (4-7).

It has been reported in literature the critical role of the muscle and ligament support in addition to the TMJ integrity in regards to the different joint functions and dynamics. For those cases where mandible resections were done with preservation of the condyle segment and hence keeping the joint capsule intact and the muscles and ligaments still attached to the condyle ramus complex, therefore, stabilizing the free end of the transplanted bone graft and contribute to initial stability of the fossa-condyle $(6,7)$, then the different TMJ functions and biomechanics were preserved or associated with insignificant changes. Only when the condyle and posterior ramus are preserved can TMJ stability be enhanced by major mandibular elevator muscles. Anyhow, the normal fossa-condyle is easily compromised after oncological mandibular reconstruction, and thus TMJ deviations should be studied in different settings to gain a better understanding and to guide reconstruction and rehabilitation of the mandible (8-10).

To rehabilitate aesthetics and function, it is important to restore a normal disc-condyle-eminence relationship, especially in the case of disarticulation resections. The disc condyle complex is important for 
the attachment of muscles and ligaments of the temporomandibular to guard against aberrant occlusion, prevent difficult chewing, asymmetric facial contours, and even pain. However, most research reports in this area only focused on studying the effects and outcomes of such dynamic and positional changes of the neocondyle segments following reconstruction of the TMJ with free fibula flaps, with no management strategy reported to avoid such sequlae $(11,12)$.

Functional movements of the human mandible during chewing and speaking have both translatory and rotatory components. For functional evaluation of the mandibular musculoskeletal system, three movements are investigated: (1) opening-closing; (2) protrusion; (3) laterotrusion. These complex movements of the mandible rely on the temporomandibular joint (TMJ) function. The terminal hinge axis is often used to describe the movements of the temporomandibular joint. Several aberrations have been noticed following mandibular resection with disarticulation of the TMJ and reconstruction with free fibula flaps ${ }^{(7)}$. These include abnormalities in dental occlusion, sagging of the mandibular condyle, abnormal mandibular movements, and disharmony of the musculoskeletal system. Such outcomes of TMJ reconstructions have not been investigated before ${ }^{(11,13-15)}$. Various alternatives for reconstructing the TMJ are being long advocated, including free grafts (costochondral, iliac crest, clavicle, or meta- tarsus), free flaps (fibula) when the defect is major, distraction osteogenesis, and alloplastic grafts, in addition to the newly introduced technique of total TMJ prosthesis $(12,13)$.

In our team, we retrospectively evaluated the outcomes of TMJ reconstruction using FFF following disarticulation resections, with the finding of sagging of the neo-mandible, changing occlusal relationship and change of the neocondyle position. As a result, we introduced three modified techniques for TMJ reconstruction following disarticulation resections using FFF as follows: (1) TMJ disc anchorage to the upper end of the fibula (neocondyle); (2) Mandible suspension using the interosseous membrane (sling suspension); (3) Muscle reattachment of lateral pterygoid and masseter muscles.

The purposes of this study are to evaluate different outcomes of following TMJ reconstruction with FFF after disarticulation resections and correlates with the different modification and factors.

\section{Materials and Methods}

An online search will be done to check all articles reporting TMJ reconstruction using FFF and advocating different outcomes. prospective clinical study was to introduce three modified techniques simultaneously with mandible reconstruction of the TMJ with vascularized free fibula flap (FFF) for dynamic mandible reconstruction and evaluate the different outcomes of such modifications in comparison to the conventional technique. 
Studies included in the systematics review

$(n=20)$

\section{Methods}

This systematic review and meta-analysis adhered to the Preferred Reporting Items for Systematic Reviews and Meta-Analyses (PRISMA) statement for reporting systematic reviews ${ }^{1}$.

\section{Focused question}

\section{Is there difference in functional outcomes between TMJ reconstruction with condyle suspension maneuvers and without?}

\section{Search strategies}

From inception to Jun 2021, an electronic search without time or language restrictions will be done in the following databases: PubMed, Scopus, and the Cochrane library (Table S2). The following terms were used in the search strategy on PubMed: ((((()(((mandible[MeSH Terms])) OR (tmj)) OR (tempromandibular joint)) OR (condyl*)) AND (disarticulation resection)) OR (mandibulectomy)) OR (condylectomy)) AND (condyle reconstruction)) OR (neocondyle)) AND (fibula free flap).

A manual search of the following journals will be also performed: Journal of oral oncology, Journal of plastic and reconstructive surgery, Journal of plastic, esthetic and reconstructive surgery, Journal of annals of plastic surgery, Journal of head and neck, Laryngoscope, British Journal of Oral and Maxillofacial Surgery, International Journal of Oral and Maxillofacial Surgery, Journal of Craniofacial Surgery, Journal of CranioMaxillofacial Surgery, Journal of Microsurgery, Oral Surgery Oral Medicine Oral Pathology Oral Radiology 
and Endodontology, Journal of head and neck surgery and JAMA otolaryngology head and neck surgery. The reference list of the identified studies and the relevant reviews on the subject will be also evaluated for possible additional studies. Moreover, online databases providing information about clinical trials in progress will be checked (clinicaltrials.gov; www. centerwatch.com/clinical trials; www.clinicalconnection.com).

\section{Inclusion and Exclusion Criteria}

This review will include all human studies (experimental or observational studies) that evaluate postoperative outcomes after reconstruction of defect involving TMJ using fibula flap. There will be no restriction on the publication date or status. (add inclusion criteria) The following outcomes will be collected: Flap failure, reoperation, readmission, and respiratory complications, delirium, sepsis, Length of hospital stays, or Hospital cost. The exclusion criteria will be other studies that reported one of the following: 1) Studies with

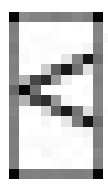

?? patients 2) Studies including patients under the age of 18,3 ) Review studies, meeting abstracts, and/or non-English articles.

\section{Data extraction process}

Two researchers independently will assess the titles, abstracts, and full-texts of the relevant studies and any controversy will be resolved by discussion to reach a consensus. The following data will be collected for each study (when available): authors, publication year, country of origin, study design, Age of the participants, number of flap failure, and other outcomes (as will be designed) (Tables 1). Two researchers independently will review the included articles and collected the data. Disagreements between the reviewers were resolved by discussion between and if necessary, a third reviewer was involved to reach a consensus. A further contact with authors for possible missing data will be also performed.

\section{Methodological quality appraisal}

Two authors independently will assess the risk of bias in the included studies. The quality assessment will be performed the Joanna Briggs Institute (JBI) Critical Appraisal tools for use in Systematic Reviews of case series $^{2}$ (Table ???). The JBI tools consisted of 10 domains and if more than 2 domains were not met, the study then considered as having a high risk of bias.

\section{References}

1. Moher D., Liberati A., Tetzlaff J., Altman DG., Group TP. Preferred Reporting Items for Systematic Reviews and Meta-Analyses: The PRISMA Statement. PLOS Med 2009;6(7):e1000097. 
2. Munn Z., Barker TH., Moola S., Tufanaru C., Stern C., McArthur A., et al. Methodological quality of case series studies: an introduction to the JBI critical appraisal tool. JBI Evid Synth 2020;18(10):2127-33. Doi: 10.11124/JBISRIR-D-19-00099.

Study flow diagram 